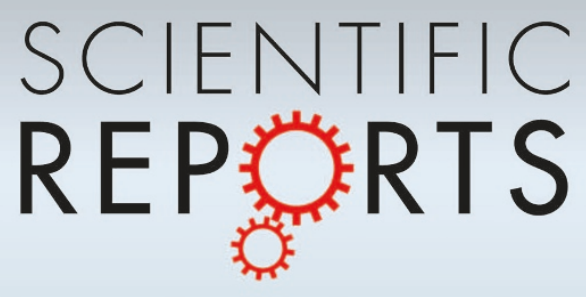

OPEN

SUBJECT AREAS:

NANOPHOTONICS AND

PLASMONICS

NONLINEAR OPTICS

OPTICAL PROPERTIES AND

DEVICES

SUB-WAVELENGTH OPTICS

Received

2 May 2013

Accepted

26 September 2013

Published

14 October 2013

Correspondence and requests for materials should be addressed to

M.K. (mkhorasa@ uwaterloo.ca) or S.S.S. (sssaini@uwaterloo.

ca)

\title{
Highly Enhanced Raman Scattering of Graphene using Plasmonic Nano-Structure
}

\author{
M. Khorasaninejad ',2, S. M. Raeis-Zadeh', S. Jafarlou', M. J. Wesolowski ${ }^{3}$, C. R. Daley33, J. B. Flannery33, \\ J. Forrest ${ }^{2,3}$, S. Safavi-Naeini' \& S. S. Saini ${ }^{1,2}$
}

\begin{abstract}
'Department of Electrical and Computer Engineering, University of Waterloo, 200 University Ave. West, Waterloo, N2L 3G1, ${ }^{2}$ Waterloo Institute of Nanotechnology, University of Waterloo, 200 University Ave. West, Waterloo, N2L 3G1, ${ }^{3}$ Department of Physics, University of Waterloo, 200 University Ave. West, Waterloo, N2L 3G1
\end{abstract}

Highly enhanced Raman scattering of graphene on a plasmonic nano-structure platform is demonstrated. The plasmonic platform consists of silver nano-structures in a periodic array on top of a gold mirror. The gold mirror is used to move the hot spot to the top surface of the silver nano-structures, where the graphene is located. Two different nano-structures, ring and crescent, are studied. The actual Raman intensity is enhanced by a factor of $\mathbf{8 9 0}$ for the G-peak of graphene on crescents as compared to graphene on a silicon dioxide surface. The highest enhancement is observed for the G-peak as compared to the 2D-peak. The results are quantitatively well-matched with a theoretical model using an overlap integral of incident electric field intensities with the corresponding intensities of Raman signals at the G-and 2D-peaks. The interaction of light with nano-structures is simulated using finite element method (FEM).

$\mathrm{n}$ recent years, remarkable linear and non-linear optical properties of graphene have been extensively demonstrated $^{1,2}$. For example, second order non-linearity using $800 \mathrm{~nm}, 150$ femtosecond laser pulses has been observed in a single-layer and multi-layer graphene $e^{3}$. It has been shown that the graphene third-order nonlinear susceptibility is one order of magnitude stronger than that of carbon nanotubes ${ }^{4}$. In addition, the bandgap of bilayer graphene can be tuned by applying external electric field ${ }^{5}$. Outstanding optical properties of graphene suit this material for a whole range of applications in optics. Recently, the photo-absorption of graphene has been used in efficient photocurrent generation with internal efficiencies in the range of $15-30 \%^{6,7}$. $\operatorname{In}^{8}$, an optical modulator was demonstrated based on tuning the Fermi level of graphene on top of a complementary metaloxide semiconductor (CMOS) compatible silicon optical waveguide. Modulation speeds of more than $1 \mathrm{GHz}$ over a broad spectrum ranging from $1.35 \mu \mathrm{m}$ to $1.6 \mu \mathrm{m}$ were achieved. Terahertz sources based on electrical or optical pumping of graphene have also been proposed ${ }^{9}$. Graphene also demonstrates non-linear optical properties such as second-harmonic generation ${ }^{3}$ and Raman scattering ${ }^{10}$ which can possibly lead to realization of optical switches and logic gates. Furthermore, since optical properties of graphene can be tuned with applying external electric field ${ }^{1}$, one can envision tunable optical components.

For many of the aforementioned optical applications, increasing light-graphene interaction can help improve the device efficiency. This concept has been considered in single emitters ${ }^{11}$ where metallic structures have been used to enhance the emission or absorption from a single emitter. Similar enhancement can be achieved by integration of graphene layers on plasmonic structures ${ }^{12,13}$. This integration allows for coupling of photons to plasmons and using the plasmon-polariton waves to interact with graphene. Very recently, plasmonic nanoantennas sandwiched between two single layers of graphene have been demonstrated for enhanced optical absorption and photodetection ${ }^{14}$. Graphene was also used as a test bed for investigating surface enhanced Raman scattering (SERS) from two closely placed gold nano-disks creating a hotspot in the gap ${ }^{15}$. The output Raman intensity from the graphene layer suspended on the nano-structure was increased by a factor of 12.8 as compared to the same layer placed on a silicon dioxide substrate. Wang et al. ${ }^{10}$ reported a large graphene Raman enhancement by a factor of $\sim 1000$ by suspending graphene on gold pyramids as compared to graphene on a gold substrate. They also measured the Raman signal of graphene on a silicon dioxide substrate and the Raman signal for graphene on silicon dioxide is at-least few times larger than graphene on gold. The enhancement achieved in ${ }^{10}$ would be smaller than the reported values if graphene on silicon dioxide is used as a reference. As we will discuss 
later, we believe that the graphene on a dielectric substrate, such as silicon dioxide, should be used as a standard test structure for comparing Raman enhancement. While enhanced Raman provides a good test bed for investigating the electric field enhancement within the graphene layer, the Raman signal can also be used for optical non-linear devices such as optical switches and logic gates as has been demonstrated for silicon waveguides and nanowires ${ }^{16,17}$.

In this paper, we demonstrate highly enhanced Raman scattering from graphene layer suspended on a plasmonic structure at an incident wavelength of $532 \mathrm{~nm}$. Using crescent nano-structures the actual output Raman intensity was increased by a factor of 890 as compared to that of graphene on silicon dioxide for the G-peak and by a factor of 38 for the 2D-peak. These results show significant enhancement as compared to $^{10}$ and $^{15}$. The plasmonic structure was optimized to achieve high intensity electric fields at the wavelength of $532 \mathrm{~nm}$ using silver nano-rings and nano-crescents on a gold mirror substrate. Since the spacing (gap) between the graphene layer and the nano-structures is not constant, the achieved enhancement is varying from one point to another point in the sample. These observations are well understood by electromagnetic modeling of these structures.

\section{Results}

Device architecture. Enhanced localized electric fields have been achieved in plasmonic structures by using small gaps ${ }^{18}$ or sharp edges ${ }^{19}$. Periodic arrangement of nano-structures also plays a crucial role in creation of localized enhancements ${ }^{20}$. In our device, we combine the sharp nano-structures in a periodic arrangement. Schematics of the proposed structures are shown in Fig. 1(a)-(b). The plasmonic configurations consist of two-dimensional periodic silver nanostructures on top of a gold-coated glass substrate. The thickness of the gold mirror was $30 \mathrm{~nm}$. Two different shapes were tested: ring and crescent. The inner and the outer diameters were varied for the both structures. The ring structure was chosen as it has previously been studied for enhanced optical properties ${ }^{21}$ while the crescent structure has sharp tips. The optimal ring structure with maximum enhancement had inner and outer diameters of $180 \mathrm{~nm}$ and $270 \mathrm{~nm}$, respectively; and the optimal crescent had a maximum width of $45 \mathrm{~nm}$ and inner and outer diameters of $130 \mathrm{~nm}$ and $220 \mathrm{~nm}$, respectively. Definition of the crescent structure can be seen in the Supplementary Information (SI). Representative scanning electron microscope (SEM) images of fabricated nanostructures are shown in Fig. 1(c)-(d). Since the electric fields are highly localized on the surface of plasmonic structure, the enhancement of the Raman signal will depend on the gap between the graphene and the nano-structures. As such, the crescent shape allows graphene to penetrate into the empty region, resulting in a smaller gap. Furthermore, the crescent structure should also create highly localized hot spots at its two corners. For both the structures, the height of the silver was $75 \mathrm{~nm}$. The nano-structures were arranged in a two dimensional square lattice with a period of $400 \mathrm{~nm}$. A unique aspect of the proposed structure is the use of a gold reflecting mirror below the silver plasmonic nano-structures. Light is reflected from the gold with a $\sim \pi$ phase shift; therefore, the hot spot locations are pushed to the top of the silver nano-structure (see the SI for the important role of the gold layer to localize the electric field on top of the nano-structure). This configuration allows for the hot spots to be in close proximity to the graphene thereby increasing the Raman intensity.

Raman measurements. Figure 2 shows the graphene Raman spectra for the two aforementioned types of nano-structures and the silicon dioxide substrate. Raman signal from the optimized structures has been plotted in Fig. 2. In addition, more measured Raman spectra are shown in the SI. Various Raman peaks are identified in this figure. The two most intense peaks are the G-peak at $\sim 1600 \mathrm{~cm}^{-1}$ and 2Dpeak at $\sim 2700 \mathrm{~cm}^{-1}$. For graphene on silicon dioxide and the ring structure, the $2 \mathrm{D}$-peak is greater than the G-peak, a signature of single layer graphene. A weak D-peak is present at $1466 \mathrm{~cm}^{-1}$ for graphene on the silicon dioxide substrate (see SI) showing that the graphene layer used in the experiment has defects to begin with. The D-peak arises due to the intra-valley double resonance process involving a single phonon and defect $^{24}$. Since we are doing relative comparisons to understand the improvement by plasmonics, these defects should not be an issue. In addition other issues such as strain
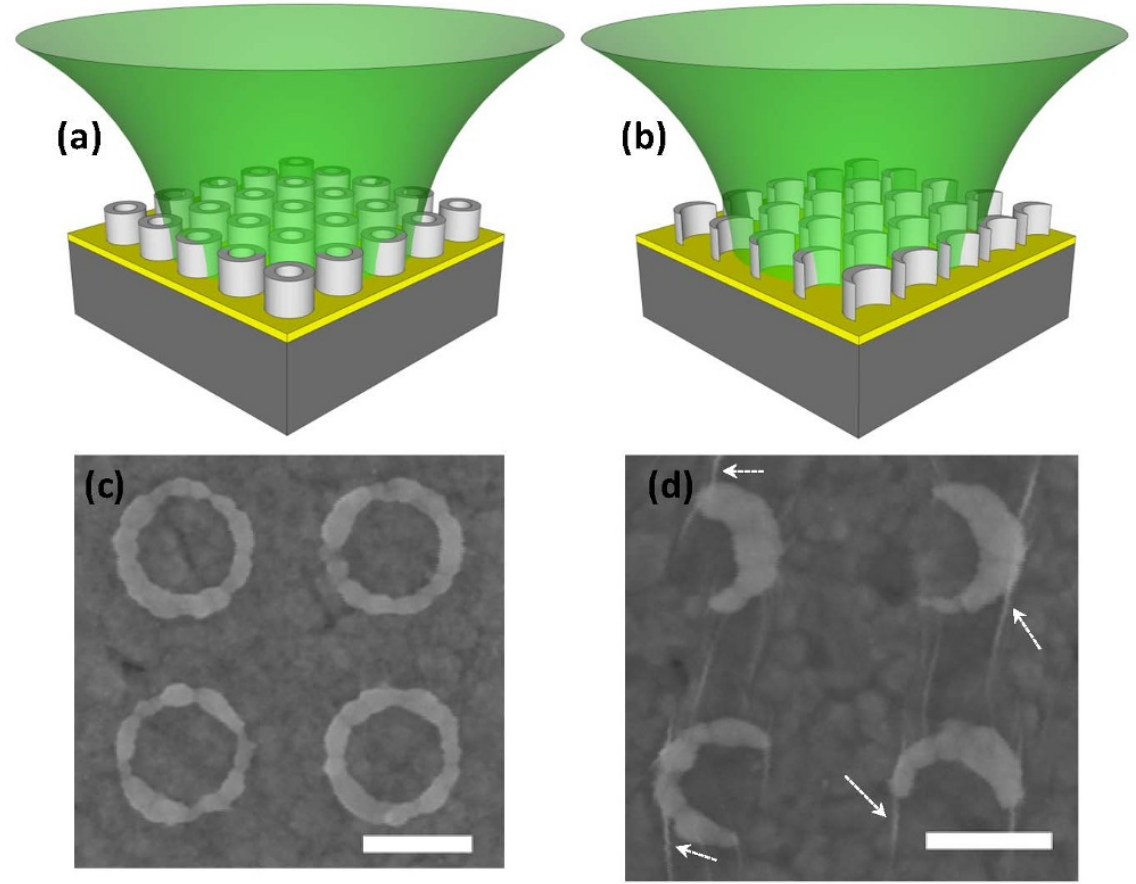

Figure $1 \mid$ (a)-(b) Schematic diagrams of ring and crescent nano-structures on a gold coated glass substrate. The thickness of gold is $30 \mathrm{~nm}$ and the height of silver nano-structures is $75 \mathrm{~nm}$. (c)-(d) Top view SEM images of graphene lies on ring and crescent nano-structures. Arrows point to the folds in the graphene on top of the crescent structure. Scale bars are $200 \mathrm{~nm}$. 


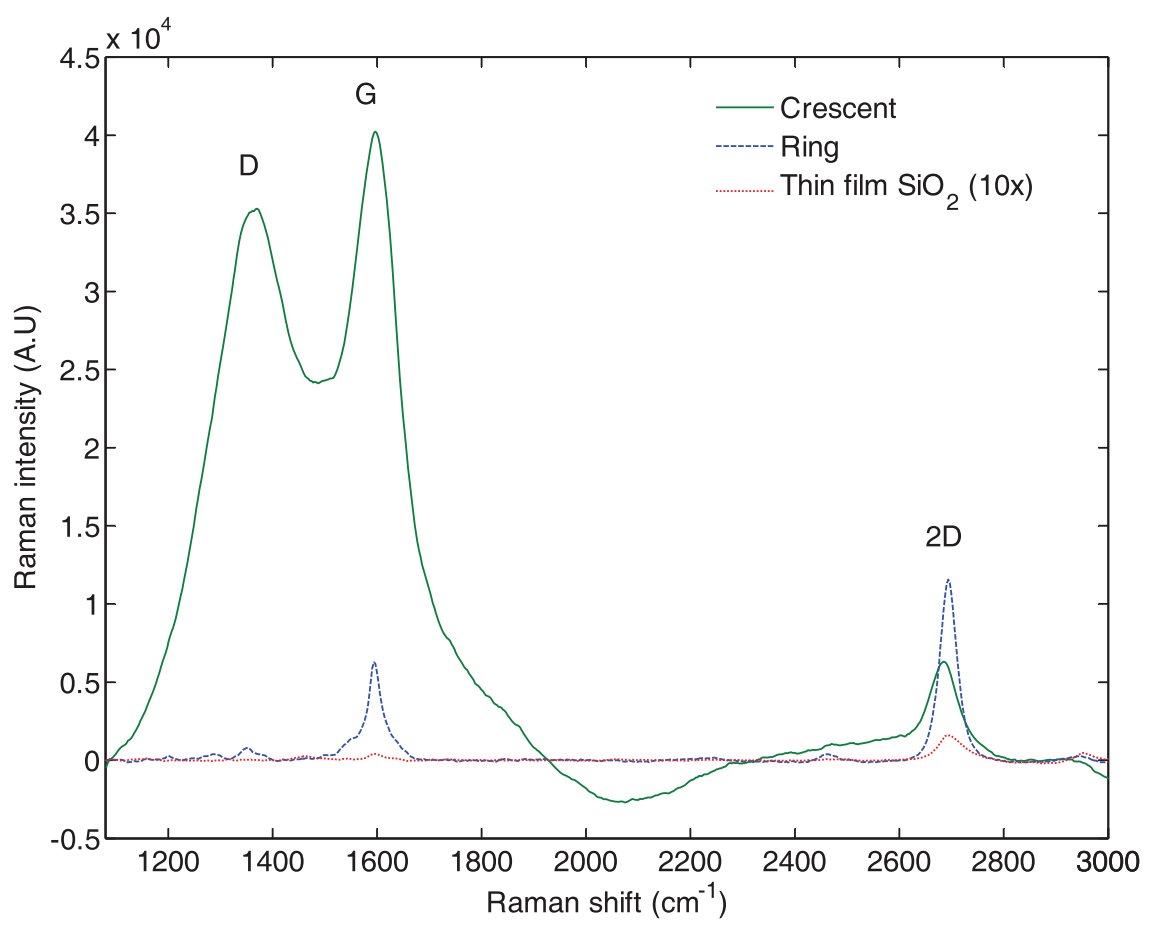

Figure 2 Raman spectra of graphene on thin film of silicon dioxide, silver rings and silver crescents. Raman spectra of graphene on thin film of silicon dioxide is multiplied by a factor of 10 for ease of visualization.

and doping of graphene can arise during and after transfer of graphene onto a non-flat substrate. The Raman signals of graphene after transfer to the nano-structures were also measured at the side of the arrays where graphene were suspended on flat gold coated substrate. No significant differences were observed over multiple measurements at different spots. For the crescent shape, D-peak at $\sim 1350 \mathrm{~cm}^{-1}$ becomes quite prominent with intensity nearly the same as that of the G-peak. This peak was weakly present in graphene on the ring structure. The origin of this peak is believed to be due to sharp graphene folds ${ }^{10}$. These folds are clearly visible in Fig. 1(d) showing the SEM image of graphene on crescents. Folds were more pronounced and present in the arrays with crescent structures as compared to the ring structures. We believe the sharp corners of the crescent structure break the symmetry of the nanostructure and cause these folds. These folds were observed in ${ }^{10}$ also which had similar sharp features in pyramids. The sharp folds may also come from the corrugations but we have not observed them in the ring nano-structure. It is to be noted that these different arrays were placed very closely together with spacing of $100 \mu \mathrm{m}$. Since, the height of our corrugation is quite small compared to the lateral dimensions and spacing between the rings, the graphene seems to have been uniformly laid over the ring nano-structure. So we believe it is not the vertical corrugation but the sharp tips which originate the folds in graphene layers. Measurements from different parts of the array always showed a large D-peak for the crescent nano-structures (see the SI).

The enhancement, the peak position and the full-width half maximum (FWHM) of the G- and 2D-peaks of all cases are summarized in Table 1. Some interesting features can be seen in the obtained results. First of all, a much larger enhancement is achieved for the G-peak as opposed to that of the 2D-peak. Second, the difference in the enhancement is much more prominent for the crescent structure as compared to that of the ring structure. For the graphene on the crescent structure, the G- and D-peaks are close together and the intensities from them overlap with each other. As such, curve fitting was used to de-convolve the Raman intensities due to these two different peaks and the enhancement was calculated from the fitted data (see the SI). For the ring and the crescent shapes, the intensity of the G-peak has been enhanced by a factor of 154 and 890, respectively. While these numbers do not change significantly for the graphene on ring structure; the enhancement is 973 if the raw experimental data is used for the graphene on the crescent structure. At the 2D-peak, the enhancements for the ring and the crescent structures are 73 and 38 , respectively. Interestingly, the enhancement for the 2D-peak for the ring geometry is almost twice as large as that of the crescent geometry. The two nano-structures have substantially different surface sizes for the silver. The difference in size of these nano-structures could also reflect in the higher Raman enhancement for crescent. However, if that was the case, enhancement would also have been higher for the 2D-peak for the crescent structure, which is not the case. Furthermore, as shown in the SI, the enhancement for the ring structure reduces as the diameter is decreased. Localized surface plasmons generated due to the periodic structure play an important role as will be discussed later.

In measuring Raman intensities from different substrates one has to be careful to make sure that graphene quality are the same as Raman signatures can vary greatly with doping, strain and growth process. The crescent and the ring structures were fabricated on the same substrate with $100 \mu \mathrm{m}$ spacing and the same graphene sample laid on them. However, because of the sharp tips of the crescent structure, the quality of the graphene on this structure is deteriorated. Result of this deterioration appears in the large measured D-peak. Broadening of the Raman signal (see table 1) also confirms the presence of defect for the crescent case. These entire defect evidences correlate well with the wrinkles observed in the SEM images in Fig. 1. This deterioration could be the reason of the 2D-peak being less than the G-peak in intensity for the crescent structure leading to a lower measured enhancement. The other issue could be the effect of doping of graphene on the gold nano-structures as compared to graphene on silicon dioxide. However, it has been shown that the $2 \mathrm{D}$ peak intensity drops as the doping level increases ${ }^{24-27}$. In our plasmonic samples, both 2D-peak and G-peak intensities have 


\begin{tabular}{|c|c|c|c|c|}
\hline Peak & Structure & Enhancement & Center Wavenumber $\left(\mathrm{cm}^{-1}\right)$ & $\mathrm{FWHM}\left(\mathrm{cm}^{-1}\right)$ \\
\hline \multirow[t]{3}{*}{ G-peak } & $\mathrm{SiO}_{2}$ & - & 1595 & 33 \\
\hline & Crescent \# 1 & 890 & 1599.5 & 140 \\
\hline & Crescent \#2 & 155 & 1596.7 & 60 \\
\hline \multirow[t]{2}{*}{ 2D-peak } & $\mathrm{SiO}_{2}$ & - & 2693.5 & 34 \\
\hline & Crescent \#2 & 31 & 2690.4 & 64 \\
\hline \multirow[t]{3}{*}{ D-Peak } & Ring & - & 1351.6 & 38 \\
\hline & Crescent \# 1 & - & 1363 & 190 \\
\hline & Crescent \#2 & - & 1361 & 155 \\
\hline
\end{tabular}

increased significantly compared to the sample on silicon dioxide which strongly suggests the role of surface plasmonic enhancement as predicted by proposed theoretical model.

To evaluate the achieved enhancement, a reasonable reference structure is needed. It is notable that, graphene layer over a gold substrate is not an appropriate reference structure because the electric field on the surface of gold vanishes (conductive boundary conditions); therefore, any nonzero finite electric field, generating even a small Raman signal, may appear as a large enhancement. We observed this problem in our samples with a minimal gap between graphene and gold where Raman signal was very weak and the enhancement appeared to be extremely large. Similarly as presented $i^{10}$, the Raman signal for graphene on gold is appreciably weaker than graphene on silicon dioxide. It is our contention that the enhancement should be evaluated with respect to that of a graphene layer on a dielectric substrate with low refractive-index such as silicon dioxide, as the reference structure. An additional advantage of such reference structure is that the reference values minimally depend on the distance between the graphene and the substrate, thus making it a reliable platform for reference. Using the graphene-on-silicon-dioxide substrate as a reference structure, a Raman enhancement of 890 for crescent and 154 for ring at the G-peak were obtained.

The spectral shape of the various Raman peaks was also examined. Lorentzian functions were fitted to the different peaks (see the SI for more details). For graphene on silicon dioxide, G- and 2D-peaks were centered at $1595 \mathrm{~cm}^{-1}$ and $2693.5 \mathrm{~cm}^{-1}$, respectively. For graphene on ring structure, both peaks were minimally shifted and were observed at $1595.4 \mathrm{~cm}^{-1}$ and $2693.4 \mathrm{~cm}^{-1}$, respectively. For the crescent structure, larger shifts were observed and the peaks were centered at $1599.5 \mathrm{~cm}^{-1}$ and $2684.5 \mathrm{~cm}^{-1}$. The shifts suggest that graphene is under strain for the crescent structure.

\section{Discussion}

As mentioned, a much higher G-peak enhancement was observed for the crescent structure. Furthermore, the enhancement behavior was different for the two structures. In order to investigate these differences, electric field distributions were calculated using HFSS (from Ansys), and are shown in Fig. 3 for the two structures at different wavelengths corresponding to the incident and the Raman signals. The $532 \mathrm{~nm}$ is the wavelength of the incident laser. Wavelengths of $582 \mathrm{~nm}$ and $622 \mathrm{~nm}$ correspond to the wavelength of Raman signals at the G-and 2D-peaks, respectively. We have previously demonstrated that silicon nanowires Raman enhancement is dependent on the overlap integral of the optical power distributions of the incident and the Raman scattered signals ${ }^{22}$. A similar approach is adopted here for graphene as follows:

$$
\text { Enhancement } \propto \frac{\int_{S}\left|E_{\lambda_{0}}\right|^{2}\left|E_{\lambda_{1}}\right|^{2} d s}{S}
$$

where $E_{\lambda o}$ and $E_{\lambda 1}$ are the electric field distributions at the input and Raman scattered wavelengths, respectively. The values of the electric field are normalized to have a power of $1 \mathrm{~W}$. For computational simulations, a planar graphene layer on top of plasmonic structures was considered. Electric field perturbations due to the presence of graphene were not modeled. Nevertheless, the simulations help us to quantify the enhancement effects. The overlap integral was done only over the planar surface, $S$, where graphene is assumed to be present. As shown in Fig. 3(a)-(b), for the crescent structure the incident field and the corresponding G-peak electric field become highly concentrated at the corners of the crescent, increasing the intensity at these points. However, the electric field at the wavelength corresponding to the $2 \mathrm{D}$-peak is not as well localized at the corners resulting in a poor overlap integral between incident and Raman signals. Thus, while giant surface plasmonic enhancements occur for the incident field and the G-peak in the structure; they do not occur at the 2D-peak. The electric field distributions for the ring structure show lesser variations with changing wavelength as compared to the crescent. Consequently, the enhancement does not decrease as much for the 2D-peak for the ring structure as it does for the crescent structure. In fact, the enhancement for the 2D-peak is larger for graphene on the ring structure as compared to that on the crescent structure. It is also found that the surface plasmonic excitations are highly sensitive to the geometrical parameters within the structure such as the height of the silver, diameter of the ring and crescent, and lattice spacing thus giving room for further enhancement. Significantly, enhancements do not increase monotonically as diameter increased; and for each structure an optimal value was achieved.

Spacing between the graphene and nano-structures also plays a major role in the achieved enhancements as the electric field is highly confined to the surface of the silver nano-structure. Atomic force microscope (AFM) measurements were conducted at different spots of the crescent sample to investigate the graphene's height relative to the substrate. The AFM images of two such spots are shown in Fig. 4 (a)-(b). Figure 4(a) shows a location where the graphene is well attached to the crescents (crescent\#1) and individual element profiles within the periodic structure can be observed. With the graphene average height of $80 \mathrm{~nm}$ and considering the height of nano-structures, $75 \mathrm{~nm}$, the average spacing between graphene and the silver top is estimated as $5 \mathrm{~nm}$. Figure 4(b) shows a place where the graphene is not well-attached to the plasmonic structures (crescent\#2) and is sitting like a tent on top of the nano-structures. In the latter, individual crescents within the periodic structure are not resolved. The average height difference in this case is $150 \mathrm{~nm}$ but it is not clear whether the graphene is touching the gold surface in middle of the crescents. Nevertheless, the distance between the graphene and silver nano-structures is obviously larger than the previous spot. Raman measurements from these two spots are shown in Fig. 4(c). The enhancement for the second spot is much weaker compared to that of the first spot for the G-peak; while 2D-peak does not decrease much. Again due to poor overlap between the distributions for the 

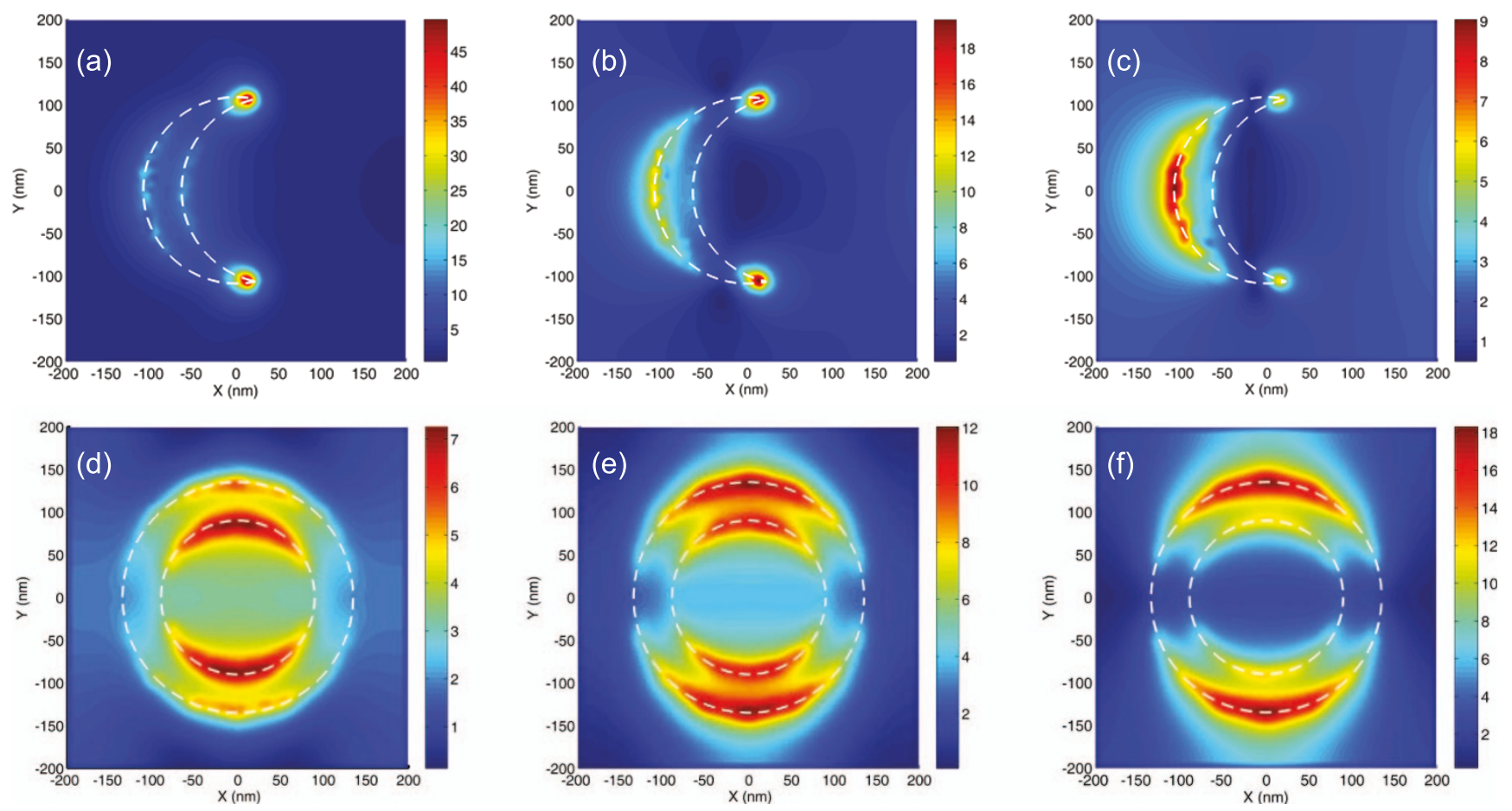

Figure 3 Electric field distribution for (a)-(c) crescent at wavelengths of $532 \mathrm{~nm}, 582 \mathrm{~nm}$ and $622 \mathrm{~nm}$ (d)-(f) ring at wavelengths of $532 \mathrm{~nm}, 582 \mathrm{~nm}$ and $622 \mathrm{~nm}$.

incident electric field and that of 2D-peak, no significant variation in intensity is expected. The sharp drop in intensity for the G-peak, as the distance increases, demonstrates the presence of strongly confined surface plasmonic fields. The FWHMs are also shown in Table 1. The FWHMs of crescent structures are still larger than those for the ring structure and silicon dioxide showing that the graphene has defects. The lower intensity for the larger gap shows that the enhancements we measure are mainly due to the plasmonic enhanced electric field. The predicted enhancements as a function of the distance between the graphene and the silver nano-structures, for both the crescent and the ring configurations are shown in Fig. 5. For the same distance between the graphene and the plasmonic structure, higher enhancement is predicted for the crescent structure, fully verifying the experimental results. Much smaller experimentally observed enhancement for the ring structures, as compared to the crescent structures, can be due to the larger gap between the graphene layer and the rings. Interestingly, the simulated difference in enhancement between the G- and 2D-peaks is less for the ring as compared to that for the crescent. As the distance between the graphene and the crescent increases, enhancement decreases more sharply for the G-peak as compared to the 2D-peak; which is again in accordance with the experimental results. The enhancement achieved is smaller than predicted by simulations which could be from the fact that the non-ideal crescent shapes were achieved experimentally. Another discrepancy observed in the simulations is the prediction of higher enhancement for the 2D-peak as opposed to G-peak for the ring structure. However, in the experiment we observed vice-versa enhancements. We simulated the structures for different fabrication tolerances and found the behavior of the G- and 2D-peak for the ring to be highly sensitive to height of the silver nano-structures. Predicted enhancements for $70 \mathrm{~nm}$ height of silver are shown in the SI and match the experimental trends. The uncertainty in height could be due to the tolerances in the measurements or possible oxidation of the silver. Furthermore, in simulations we consider ideal crescents with sharp edges while the fabricated structures were rounded off. Nevertheless, the simulations do help understand the enhancement and especially the difference G and 2D peaks for the two different structures.

As shown by simulations, the reduction of the Raman intensity with increased spacing correlates well with the AFM measurements. Not only is the reduction predicted but also the different behavior for the G-and 2D-peak for different spacing is also shown. These results also demonstrate that the optoelectronic devices made of graphene on plasmonic structures can be directly characterized using simple Raman measurements. In addition, the Raman spectroscopy can effectively be used to investigate the impact of graphene folds, difference in the heights etc. minimizing the need for more time consuming characterization methods such as AFM or SEM.

In summary, we have demonstrated three orders of magnitude of Raman enhancement from a monolayer of graphene using silver plasmonic nano-structures placed on the gold mirror. AFM measurement revealed critical role of graphene topography over the nano-structure in intensifying light-graphene interactions. In addition, a FEM simulation model was developed to understand the lightnano-structure-graphene interactions and its effects on Raman enhancement of graphene at two different peaks. This extremely enhanced light-graphene interaction will find potential applications in several areas including solar cells, photodetectors, optical modulators and nonlinear optical devices.

\section{Methods}

Fabrication. First, $5 \mathrm{~nm}$ of titanium followed by $30 \mathrm{~nm}$ of gold was deposited on a Corning glass wafer using electron beam deposition. Then, the sample was spin coated with PMMA-A4 at speed of $3000 \mathrm{rpm}$ resulting in a $190 \mathrm{~nm}$ thick layer of PMMA. The sample was baked in a vacuum oven for 20 minute at $180^{\circ} \mathrm{C}$. Electron beam lithography was carried out using a Raith TWO-150 at $25 \mathrm{KV}$. The pattern was developed on MIBK:IPA $1: 3$ bath for $40 \mathrm{~s}$ followed by $30 \mathrm{~s}$ rinsing with IPA. Next, $75 \mathrm{~nm}$ thick silver was deposited and then the sample was soaked in PG remover over night to accomplish the lift-off process. The graphene was purchased from ACS Material $^{23}$. The upper side of the graphene monolayer is covered with $500 \mathrm{~nm}$ of PMMA and the PMMA-graphene film is laid on a polymer substrate. To transfer the graphene, first the substrate was immersed in deionized (DI) water which results in releasing and then floating the PMMA-graphene film on the water surface. Subsequently the PMMA-graphene film was scooped out of water using the plasmonic chip. After the transfer, droplets of water were observed to be trapped 

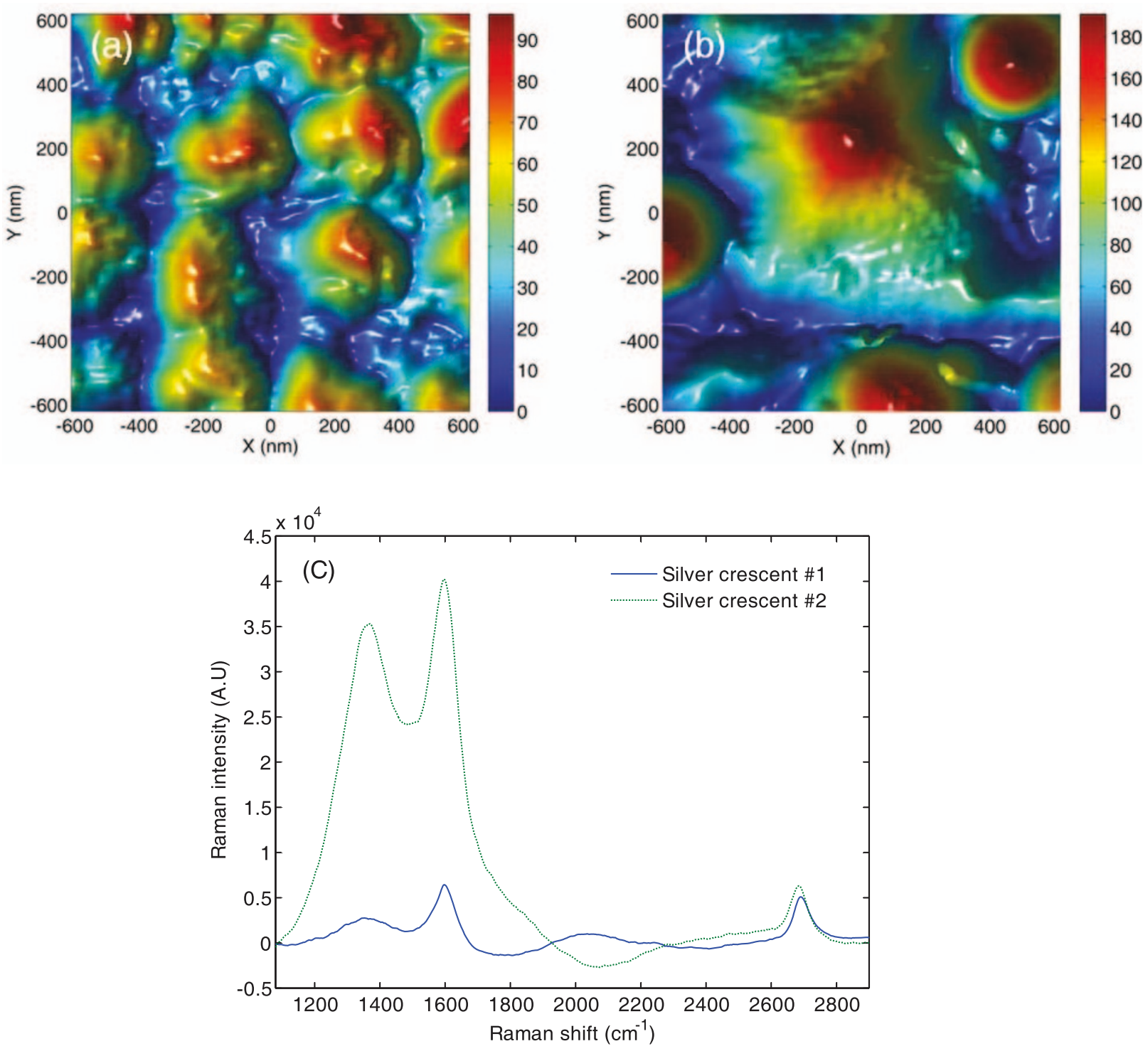

Figure 4 AFM images of two different spots: (a) silver crescent \#1 (b) and silver crescent \#2 showing how graphene lies on crescent nano-structures. (c) Raman spectra measured for these two different spots.
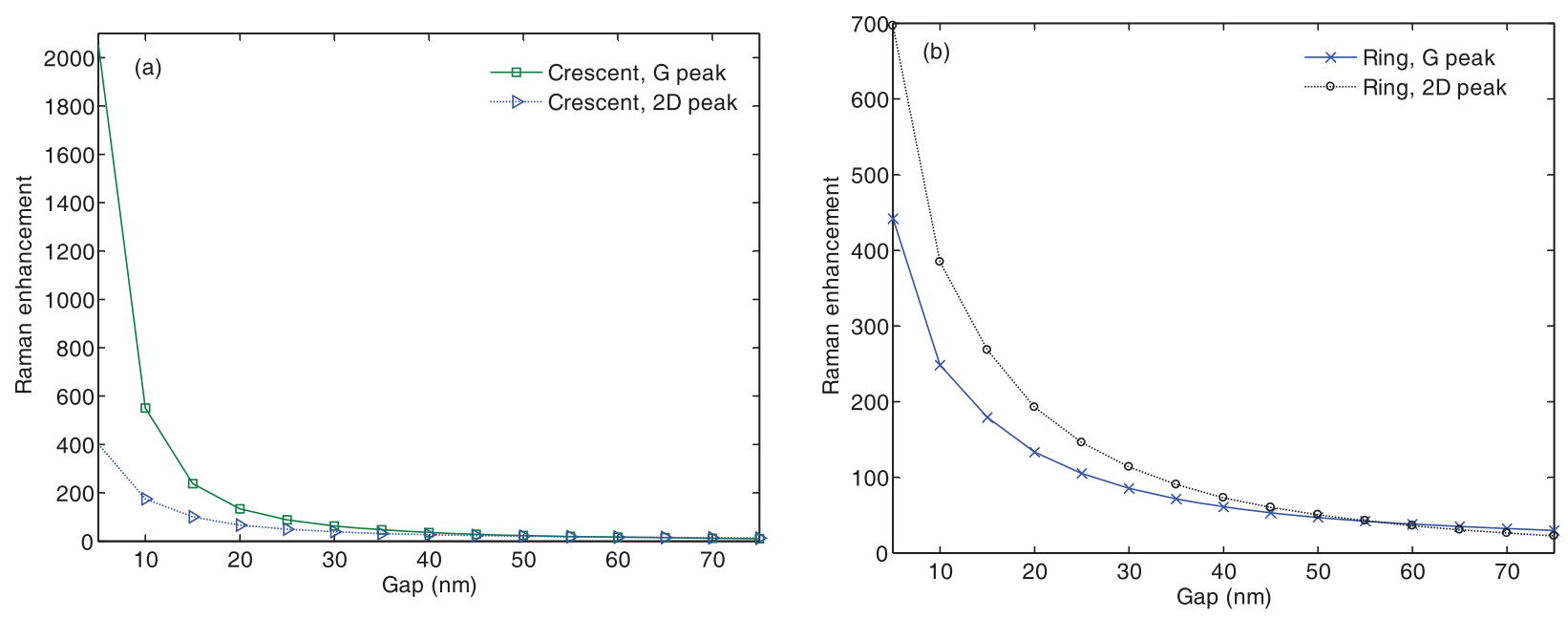

Figure 5 | Simulated enhancement of Raman for (a) crescent and (b) ring nano-structures at two different Raman peaks of graphene. 
between the array of nano-structures and the graphene layer. To dehydrate the sample, the graphene was blown by a nitrogen gun for a few minutes and then heated in an oven. Due to these trapped water droplets and some wrinkles that developed during the transfer process, the graphene tends to lie differently on the ring and crescent shaped nano-structures. Finally, the sample was soaked in acetone to dissolve the PMMA and make the sample ready for Raman measurements.

Raman measurements. The Raman spectra of graphene were measured at several spots on each array, using a Horiba Jobin Yvon HR800 series micro-Raman spectrometer coupled to an Olympus inverted microscope. A laser beam with a wavelength of $532 \mathrm{~nm}$ was focused onto the sample with a $20 \times$ short working distance objective that had a numerical aperture of 0.40 , resulting in a spot size of $\sim 1 \mu \mathrm{m}$ in diameter. The laser power was $1 \mathrm{~mW}$ and spectra were recorded with an exposure time of $15 \mathrm{~s}$ and summed over 10 accumulations.

Atomic force microscopy. AFM measurements of the graphene surface were acquired using a JPK Nanowizard 3 AFM operating in intermittent-contact mode. Scans were performed with a Budget Sensors TAP-150 G intermittent contact cantilever; nominally, spring constant of $k=5 \mathrm{~N} / \mathrm{m}$, resonance frequency of $f=$ $150 \mathrm{kHz}$.

1. Bonaccorso, F., Sun, Z., Hasan, T. \& Ferrari, A. C. Graphene photonics and optoelectronics. Nature Photon. 4, 611-622 (2010).

2. Bao, Q. \& Loh, K. P. Graphene photonics, plasmonics, and broadband optoelectronic devices. ACS Nano. 6, 3677-3694 (2012).

3. Dean, J. J. \& van Driel, H. M. Second harmonic generation from graphene and graphitic films. Appl. Phys. Lett. 95, 261910 (2009).

4. Hendry, E., Hale, P. J., Moger, J. J., Savchenko, A. K. \&. Mikhailov S. A. Strong nonlinear optical response of graphene flakes measured by four-wave mixing. Preprint at http://arxiv.org/abs/0912.5321vl, (2009), accessed: Aug. 28 ${ }^{\text {th }}, 2013$.

5. Zhang, Y. et al. Direct observation of a widely tunable bandgap in bilayer graphene. Nature. 459, 820-823 (2009).

6. Park, J., Ahn, Y. H. \& Ruiz-Vargas, C. Imaging of photocurrent generation and collection in single-layer graphene. Nano Lett. 9, 1742-1746 (2009).

7. Xia, F. et al. Photocurrent imaging and efficient photon detection in a graphene transistor. Nano Lett. 9, 1039-1044 (2009).

8. Liu, M. et al. A graphene-based broadband optical modulator. Nature 474, 64-67 (2011)

9. Otsuji, T. et al. Observation of amplified stimulated terahertz emission from optically pumped epitaxial graphene heterostructures. Preprint at $<\mathrm{http}: / /$ arxiv. org/abs/1001.5075vl> (2010), accessed: Aug. $28^{\text {th }}, 2013$

10. Wang, P. et al. Giant optical response from graphene-plasmonic system. ACS Nano. 6, 6244-6249 (2012).

11. Zumofen, G., Mojarad, N. M., Sandoghdar, V. \& Agio, M. Perfect Reflection of light by an oscillating dipole. Phys. Rev. Lett. 101, 180404 (2008).

12. Echtermeyer, T. J. et al. Strong plasmonic enhancement of photovoltage in graphene. Nature Commun. 2, 458 (2011).

13. Grigorenko, A. N., Polini, M. \& Novoselov, K. S. Graphene plasmonics. Nature Photon. 6, 749-758 (2012).

14. Fang, Z. et al. Graphene-antenna sandwich photodetector. Nano Lett. 12, 3808-3813 (2012).

15. Heeg, S. et al. Polarized plasmonic enhancement by Au nanostructures probed through Raman scattering by suspended graphene. Nano Lett. 13, 301-308 (2013)

16. Claps, R., Dimitropoulos, D., Raghunathan, V., Han, Y. \& Jalali, B. Observation of stimulated Raman amplification in silicon waveguides. Opt. Express. 11, $1731-1739$ (2003)
17. Khorasaninejad, M. \& Saini, S. S. All optical logic gates using nonlinear effects in silicon on insulator waveguides. Appl. Opt. 48, F32-F37 (2009).

18. Zhu, W., Banaee, M. G., Wang, D., Chu, Y. \& Crozier, K. B. Lithographically fabricated optical antennas with gaps well below $10 \mathrm{~nm}$. Small 7, 1761-1766 (2011).

19. Lu, Y., Liu, G. L., Kim, J., Mejia, Y. X. \& Lee, L. P. Nanophotonic crescent moon structures with sharp edge for ultrasensitive biomolecular detection by local electromagnetic field enhancement effect. Nano Lett. 5, 119-124 (2005).

20. Barnes, W. L., Dereux, A. \& Ebbesen, T. W. Surface plasmon subwavelength optics. Nature 424, 824-830 (2003).

21. Aizpurua, J. et al. Optical Properties of Gold Nanorings. Phys. Rev. Lett. 90, 057401 (2003).

22. Khorasaninejad, M., Walia, J., Dhindsa, N. \& Saini, S. S. Highly enhanced Raman scattering from vertical silicon nanowire arrays. Appl. Phys. Lett. 101, 173114 (2012).

23. ACS Material-Advanced Chemicals Supplier http://acsmaterial.com/product. asp?cid $=968$ id $=118$ accessed: Aug. $28^{\text {th }}, 2013$.

24. Basko, D. M., Piscanec, S. \& Ferrari, A. C. Electron Electron Interactions and Doping Dependence of the Two-Phonon Raman Intensity in Graphene. Phys. Rev. B. 80, 165413 (2009).

25. Das, A. et al. Monitoring dopants by Raman scattering in an electrochemically top-gated graphene transistor. Nature Nanotech. 3, 210-215 (2008).

26. Casiraghi, C., Pisana, S., Novoselov, K. S., Geim, A. K. \& Ferrari, A. C. Raman fingerprint of charged impurities in graphene. Appl. Phys. Lett. 91, 233108 (2007).

27. Casiraghi, C. Doping Dependence of the Raman Peaks Intensity of Graphene close to the Dirac Point. Phys. Rev. B. 80, 2333407 (2009).

\section{Acknowledgments}

This work was generously supported by the Natural Sciences and Engineering Research Council of Canada (NSERC) and BlackBerry (formerly Research in Motion). The infrastructure used for this work would not be possible without the significant contributions of the Canada Foundation for Innovation, the Ontario Ministry of Research \& Innovation and Industry Canada. Their support is gratefully acknowledged.

\section{Author contributions}

M.K. conceived the idea, designed the experiments, and fabricated the devices. S.M.R. and S.J. carried out the simulations. M.J.W. and B.J.F. did the Raman measurements. C.R.D. did the AFM measurements. J.F., S.S.-N. and S.S.S. took part in the data interpretation and discussions. The work was supervised by S.S.S.

\section{Additional information}

Supplementary information accompanies this paper at http://www.nature.com/ scientificreports

Competing financial interests: The authors declare no competing financial interests.

How to cite this article: Khorasaninejad, M. et al. Highly Enhanced Raman Scattering of Graphene using Plasmonic Nano-Structure. Sci. Rep. 3, 2936; DOI:10.1038/srep02936 (2013).

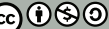

This work is licensed under a Creative Commons Attribution-

NonCommercial-ShareAlike 3.0 Unported license. To view a copy of this license, visit http://creativecommons.org/licenses/by-nc-sa/3.0 